\title{
Composición y aplicaciones clínicas de especies de chiles (Capsicum spp.) domesticados
}

\section{Composition and clinical applications of domesticated chili pepper (Capsicum spp.) species}

\author{
Lorena M. Osorio-Barraza ${ }^{a}$, Salvador Manzur-Valdespino ${ }^{b}$, Quinatzin Y. Zafra-Rojas ${ }^{c}$, Luis \\ Delgado-Olivares ${ }^{d}$, Zuli G. Calderón-Ramos ${ }^{e}$ Nelly del S. Cruz-Cansino ${ }^{f}$
}

\begin{abstract}
:
The Capsicum genus originated in Latin America and spread to other continents through the exchange of spices. Its fruit is used fresh or dried to add colour, flavour and smell to dishes, salads and sauces. It has great commercial relevance worldwide, being the main producing countries China, Mexico and Turkey. There are five domesticated species, which are: C. baccatum, $C$. chinense, $C$. pubescens, $C$. frutescens and $C$. annuum. In general, the fruits of Capsicum have a great variety of sizes and shapes, and according to their state of maturity they present changes in the tonalities, which go from green to yellow, red and intense red tones. The fruits have bioactive compounds that can provide beneficial effects on health. The objective of the present work was to perform a review about the bioactive compounds of the different domesticated species of the genus Capsicum and its recent clinical applications. Due to the bioactive components of Capsicum species, studies show beneficial effects in the prevention and treatment of non-communicable diseases, anti-inflammatory effects, weight control, cardiovascular, anti-diabetic, gastrointestinal and carcinogenic effects. Capsicum species have a prominent role in the medical field due to their ability to modulate and modify several processes at biological
\end{abstract}

Keywords:

Capsicum, Chili, bioactive compounds, clinical applications

\section{Resumen:}

El género Capsicum es originario de América Latina y se expandió a otros continentes mediante intercambio de especias. Su fruto se utiliza fresco o seco para aportar color, sabor y olor a platillos, ensaladas y salsas. Posee gran relevancia comercial a nivel mundial, siendo los principales países productores China, México y Turquía. Existen cinco especies domesticadas que son: $C$. baccatum, $C$. chinense, $C$. pubescens, $C$. frutescens y $C$. annuum. En general, los frutos de Capsicum disponen de gran variedad de tamaños y formas, y de acuerdo a su estado de madurez presentan cambios en las tonalidades, que van del verde a tonos amarillos, rojos y rojos intensos. Los frutos poseen compuestos bioactivos que pueden proveer efectos benéficos en la salud. El objetivo del presente trabajo fue realizar una revisión sobre los compuestos bioactivos de las diferentes especies domesticadas del género Capsicum y sus recientes aplicaciones clínicas. Debido a los componentes bioactivos de las especies de Capsicum, los estudios demuestran efectos benéficos en la prevención y tratamiento sobre enfermedades no transmisibles, efectos antiinflamatorios, control de peso, cardiovasculares, antidiabéticos, gastrointestinales y cancerígenos. Las especies de Capsicum tienen un notable papel en el campo médico debido a su capacidad de modular y modificar diversos procesos a nivel biológico.

\footnotetext{
a Universidad Autónoma del Estado de Hidalgo, https://orcid.org/0000-0001-5162-054X, Email: os325538@uaeh.edu.mx

b Universidad Autónoma del Estado de Hidalgo, https://orcid.org/0000-0003-2447-0413, Email: salvador_manzur@uaeh.edu.mx

c Universidad Autónoma del Estado de Hidalgo, https://orcid.org/0000-0002-5295-9972, Email: quinatzin_zafra@uaeh.edu.mx

d Universidad Autónoma del Estado de Hidalgo, https://orcid.org/0000-0002-3506-8393, Email: 1delgado@uaeh.edu.mx

e Universidad Autónoma del Estado de Hidalgo, https://orcid.org/0000-0002-6367, Email: zramos@uaeh.edu.mx

Autor de Correspondencia, Universidad Autónoma del Estado de Hidalgo, https://orcid.org/0000-0002-6771-3684, Email:
} ncruz@uaeh.edu.mx 


\section{Palabras Clave:}

Capsicum, chile, compuestos bioactivos, aplicaciones clínicas

\section{Introducción}

Los chiles, ajíes o pimientos, son nombres utilizados coloquialmente para referirse a las especies del género Capsicum, pertenecientes a la familia de las solanáceas. ${ }^{1,2}$ Está integrado por 30 especies de chile, de las cuales 5 han sido domesticadas $(C$. annuum L., $C$. chinense jacq., $C$. frutescens $L$., $C$. baccatum $L$. y $C$. pubescens Ruiz \& Pav.) y distribuidas en todo el mundo, destacando el $C$. annuum como la de mayor superficie cultivada y producción por hectárea. ${ }^{3}$ Esta especie es originaria de los trópicos de América Latina ${ }^{4}$ y se introdujo a otros continentes mediante intercambios comerciales de especias. ${ }^{5,6}$ China, México y Turquía son los principales productores de esta especie; la producción anual en el 2018 fue de 18, 3 y 2 millones de toneladas, respectivamente, de acuerdo a información de la Organización de las Naciones Unidas para la agricultura y la alimentación ( $F A O$, por sus siglas en inglés: Food and agriculture organization of the United Nations). ${ }^{7}$ En la cocina internacional, el chile se utiliza en general para aportar color, olor y sabor picante a los platillos. ${ }^{8}$ Este fruto se utiliza principalmente en la industria de la pasta, y la producción de especias y en forma fresca, en rodajas o en polvo para la preparación de guisos y sopas, así como para la elaboración de platillos, asados y salsas. ${ }^{9}$ Los frutos poseen compuestos bioactivos, como fenoles, flavonoides, carotenoides y capsaicinoides siendo estos últimos los compuestos representativos ya que aportan la pungencia o sensación de ardor característica. ${ }^{10}$ Estas especies tienen importante uso gastronómico, sin embargo, se ha aplicado en el ámbito clínico contra enfermedades no transmisibles, procesos inflamatorios y cáncer, entre otros, debido al contenido de componentes que le confieren efectos benéficos para la salud. ${ }^{11}$

El objetivo del presente trabajo fue recopilar información sobre los compuestos bioactivos de las diferentes especies domesticadas del género Capsicum y los estudios más recientes sobre aplicaciones clínicas.

\section{Morfología y composición de las especies domesticadas del género Capsicum}

La variabilidad que se presenta entre las especies del género Capsicum está relacionada con la procedencia y las condiciones climáticas, que son factores que influyen de manera significativa. ${ }^{12}$ Debido a las variaciones en cuanto a forma, tamaño y color de los frutos, estas especies se definen de acuerdo a las características de las estructuras florales. ${ }^{6}$

Dentro de los chiles, la especie $C$. annuum, presentan la mayor diversidad de color, tamaño y forma. Estos frutos inmaduros son verdes, amarillos, blancos y en su estado maduro presentan colores rojos, naranjas y marrones (figura 1A); presentan formas redondas, alargadas, cónicas, acampanadas o ligeramente aplanados, pueden llegar a medir entre $0.5 \mathrm{~cm}$ y $43 \mathrm{~cm}$ de largo ${ }^{6}$ su peso promedio en estado fresco es de $6.9 \mathrm{~g}$. Contienen semillas de color amarillo pálido. ${ }^{1,13} \mathrm{El}$ número de semillas varía ampliamente dependiendo del fruto. Sin embargo, en especies como Capsicum annuum var. glabriusculum se ha reportado hasta 41 semillas. ${ }^{12}$

Los frutos de la especie $C$. baccatum en su estado inmaduro presentan coloraciones verdes y en su estado maduro, predomina el color rojo, seguido de tonos naranja y amarillo (figura 1B). Una variedad conocida como "ají cereza" mide en promedio $1.24 \mathrm{~cm}$ de longitud; sin embargo, la forma de los frutos cambia de acuerdo a la variedad y estos pueden ser alargados y cuadrados, su peso promedio es de $7.5 \mathrm{~g}$ y su semilla es de color amarillo claro. ${ }^{10,13}$

La especie $C$. chinense es de pulpa firme, presenta forma de trompo con áreas hundidas. Sus frutos son de color verde esmeralda en estado inmaduro hasta alcanzar color anaranjado brillante en estado de madurez (figura 1C), miden de $3 \mathrm{~cm}$ a $5 \mathrm{~cm}$ de longitud y pesan de $6 \mathrm{~g}$ a 10 $g^{10,14}$, el número de semillas en una especie de $C$. chinense conocida como "ají mochero" es de 16 semillas por fruto. ${ }^{15}$ Los frutos de la especie $C$. frutescens tienen una longitud y peso de $2.2 \mathrm{~cm}$ a $4.1 \mathrm{~cm}$ y de $1 \mathrm{~g} \mathrm{a} 2.30 \mathrm{~g}$, respectivamente, son alargados, de ápice puntiagudo (figura 1D), esta especie contiene entre 15 y 16 semillas de color amarillo por fruto. ${ }^{16}$ Los frutos de C. pubescens son de color amarillo, naranja y rojo, generalmente son redondos o en forma de campana y levemente corrugados (figura 1E), pueden medir entre $3.5 \mathrm{~cm}$ y $7.7 \mathrm{~cm}$ de ancho y tienen entre 45 y 100 semillas por fruto, de coloración negra. ${ }^{17}$ 


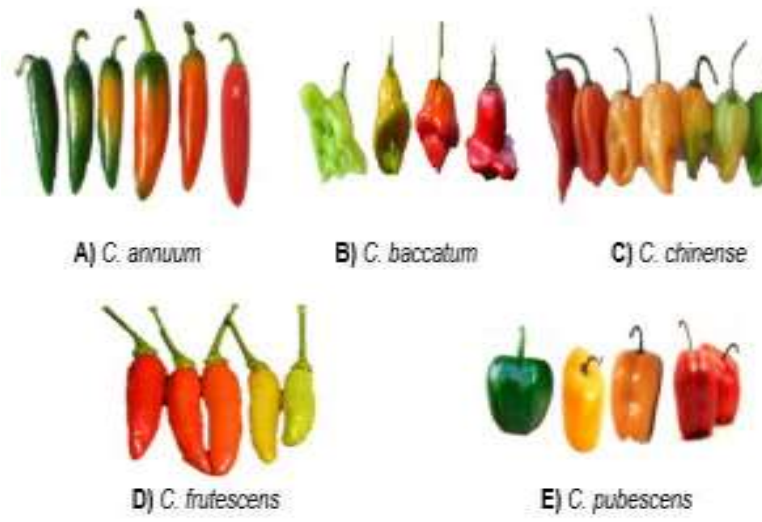

Figura 1. Morfología y diferentes estados de madurez en especies de Capsicum

Figure 1. Morphology and different maturity in Capsicum species

La composición nutrimental de los chiles puede cambiar dependiendo de la especie y variedad, así como la composición de compuestos bioactivos, los cuales están influenciados por diversos factores, como la etapa de maduración, el ambiente, el cultivo, el genotipo o la locación geográfica. ${ }^{18}$

\section{Compuestos bioactivos}

Los chiles poseen variedad de metabolitos activos, los cuales aportan sabores y colores característicos. Los compuestos fenólicos, actúan como compuestos antioxidantes protegiendo a las células contra el daño oxidativo, algunos de ellos son el ácido gálico, ácido ferúlico y ácido clorogénico. ${ }^{19}$ Las variaciones en el contenido de compuestos fenólicos en diferentes especies del género Capsicum, se deben a las diferencias genéticas de cada tipo de fruto. ${ }^{20}$ También contienen flavonoides que son compuestos que protegen a las células de los radicales libres. ${ }^{21}$ Por otra parte, los chiles presentan pigmentos que confieren color al fruto como los carotenoides, $\alpha$-carotenos, $\beta$-carotenos y capsantinas, los cuales dan la coloración roja o naranja (estado de madurez total), mientras que las clorofilas y xantofilas brindan una coloración verde (estado inmaduro). ${ }^{22}$

Como se puede observar en la Tabla $1, C$. baccatum tiene mayor contenido de fenoles totales, $C$. chinense en flavonoides, carotenoides y capsaicina, mientras que $C$. annuum en capsantina. Las diferencias en el contenido de estos compuestos, se relacionan con la etapa de maduración, métodos de extracción, genotipo y medio ambiente, así como de la variedad de frutos que contiene cada especie. ${ }^{29}$ Además, estos frutos presentan compuestos llamados capsaicinoides, son amidas formadas por la unión de la vainillilamina con un ácido graso. Se ha establecido que más del $90 \%$ de estos capsaicinoides están representados por la capsaicina y la dihidrocapsaicina, los cuales, al consumirlos son los responsables de dar la característica de sensación de dolor $\mathrm{o}$ ardor y sucede cuando las capsaicinas se unen a los receptores vaniloides-VR (TRPV) ${ }^{30}$, estos compuestos se producen y acumulan en el tejido placentario de los frutos..$^{31}$ La presencia de estos compuestos va a depender del cultivo, irrigación o estado de madurez. ${ }^{30} \mathrm{El}$ contenido de capsaicina es variable, encontrando valores de entre $532.85 \mathrm{mg} / \mathrm{kg}$ y $12473.44 \mathrm{mg} / \mathrm{kg}$, dependiendo la especie y tipo de fruto (Tabla 1). De manera general, los compuestos mencionados anteriormente, tienen actividad antioxidante, analgésica, antiinflamatoria y antibacteriana, por lo que se han utilizado ampliamente en el área médica. 22, 32

\section{Aplicaciones clínicas}

\section{Efecto antiinflamatorio}

La aplicación de una pomada con $2 \mathrm{mg}$ de carotenoides ( $2 \mathrm{~g} / \mathrm{g}$ pomada) de pimienta cayena (C. annuum) en extremidades de ratas Wistar con artritis inducida, redujo del $20 \%$ al $50 \%$ el edema en el área de inflamación, además de que las células blancas disminuyeron en el área de inflamación en el día 10 de tratamiento. ${ }^{33} \mathrm{La}$ administración de $400 \mathrm{mg} / \mathrm{kg}$ de extracto de hierbas con C. frutescens en ratas Wistar con edema inducido, disminuyó $31.4 \%$ el volumen de edema en extremidades, similar al efecto obtenido con indometacina (fármaco antiinflamatorio de referencia) (31.2\%), el extracto redujo $54.1 \%$ los niveles de proteínas en el exudado, disminuyó el $60.2 \%$ la migración de leucocitos y el 69 \% la migración de monocitos. ${ }^{34}$ Por otro lado, la aplicación de $320 \mu \mathrm{g} / \mathrm{mL}$ de extractos de oleorresina de Capsicum, disminuyeron los niveles de óxido nítrico producidos por lipopolisacáridos (LPS) en macrófagos murinos RAW 264.7, produciendo $8.2 \%$ de óxido nítrico, además, el extracto en dosis de $50 \mu \mathrm{g} / \mathrm{mL}$ disminuyó la expresión de la enzima óxido nítrico sintasa (iNOS). ${ }^{35}$ En ensayos in vitro con células murinas, $100 \mu \mathrm{g} / \mathrm{mL}$ de extractos metanólicos de 2 variedades de Capsicum. tuvieron la capacidad de reducir la producción de óxido nítrico en las células estimuladas con lipopolisacáridos. ${ }^{36}$ Por otro lado, $100 \mu \mathrm{g}$ de extractos metanólicos de frutos de $C$. annuum inhibieron la formación de edema inducido en extremidades de ratones hasta en un $40 \%{ }^{37}$

Los estudios antes mencionados sobre la actividad antiinflamatoria con la aplicación del Capsicum, reportan que se atribuye al contenido de compuestos fenólicos y flavonoides los cuales eliminan radicales libres $^{36}$, ya que tienen efecto antioxidante sobre los intermediarios reactivos de oxígeno y radicales peróxidos, que son producidos durante el proceso inflamatorio. ${ }^{33}$ 
Tabla 1. Compuestos bioactivos de las especies de Capsicum ${ }^{A}$

Table 1. Bioactive compounds of Capsicum species ${ }^{A}$

\begin{tabular}{|c|c|c|c|c|c|c|}
\hline Especie & $\begin{array}{c}\text { Fenoles } \\
\text { Totales } \\
\end{array}$ & Flavonoides & Carotenoides & Capsantina & Capsaicina & Referencia \\
\hline C. annuum & $\begin{array}{c}9211 \text { a } 11 \\
571^{\mathrm{a}}\end{array}$ & $\begin{array}{c}6541 \text { a } 20 \\
576^{\mathrm{a}}\end{array}$ & $\begin{array}{l}199.32 \mathrm{a} \\
213.87^{\mathrm{b}}\end{array}$ & $16332^{\mathrm{c}}$ & 2672 a $3289^{b}$ & $10,23,24$ \\
\hline $\begin{array}{c}C . \\
\text { baccatum }\end{array}$ & $\begin{array}{c}14928 \text { a } 18 \\
751^{d}\end{array}$ & $\begin{array}{l}34.36 \mathrm{a} \\
102.48^{\mathrm{d}}\end{array}$ & 8.75 a $25.44^{b}$ & $\begin{array}{c}4486 \text { a } 12 \\
923^{c}\end{array}$ & 827.13 a $1454.66^{b}$ & $23,25,10$ \\
\hline C. chinense & $\begin{array}{c}6392 \text { a } 12 \\
115^{\mathrm{a}}\end{array}$ & $\begin{array}{c}7056 \text { a } 23 \\
110^{\mathrm{a}}\end{array}$ & $\begin{array}{l}99.47 \mathrm{a} \\
350.12^{b}\end{array}$ & $104^{c}$ & $\begin{array}{c}10541.88 \text { a } 12 \\
473.44^{b}\end{array}$ & $10,24,26$ \\
\hline $\begin{array}{c}C . \\
\text { frutescens }\end{array}$ & $\begin{array}{c}6691 \text { a } 11 \\
189^{\mathrm{a}}\end{array}$ & $\begin{array}{c}7433 \text { a } 7 \\
944^{\mathrm{a}}\end{array}$ & $\begin{array}{c}126.99 a \\
143.27^{b}\end{array}$ & $14434^{c}$ & $\begin{array}{c}1530.74 \text { a } 2 \\
662.55^{\mathrm{b}}\end{array}$ & $27,28,10,24$ \\
\hline $\begin{array}{c}\text { C. } \\
\text { pubescens }\end{array}$ & 712 a $822^{\mathrm{e}}$ & $\begin{array}{c}256 \mathrm{a} \\
334.63^{\mathrm{e}}\end{array}$ & 9.31 a $24.09^{b}$ & $\begin{array}{c}5713 \text { a } 7 \\
895^{c}\end{array}$ & 532.85 a $647.35^{b}$ & 23,10 \\
\hline
\end{tabular}

valores corresponden a frutos en etapa madura

Del mismo modo, estos compuestos actúan sobre enzimas moduladoras de la inflamación que a su vez modifican la expresión de moléculas pro inflamatorias, como el óxido nítrico y el factor de necrosis tumoral-a. ${ }^{38}$

\section{Control de peso y actividad hipolipemiante}

En ratones C57BL/6 con obesidad inducida por una dieta alta en grasas, la ingesta de $2.39 \mathrm{~g} / \mathrm{d}$ de una fórmula con $0.05 \mathrm{~g}$ de extracto de capsaicinoides (C. annuum) fermentados, tuvo la capacidad de evitar el aumento de peso en un $20 \%$; disminuyó $(p<0.05)$ los niveles de colesterol y triglicéridos en hígado, suero y heces fecales, también redujo la cantidad de tejido adiposo, comparado con ratones alimentados con una dieta alta en grasa sin capsaicinoides. Los efectos se debieron a que los extractos mejoraron la sensibilidad de las hormonas leptina (hormona que produce saciedad) y grelina (hormona estimula apetito), donde se aumentaron y disminuyeron los niveles plasmáticos de estas hormonas, respectivamente, además aumentó la expresión de PPAR-y, C/EBPa (reguladores de adipocitos), y regularon la expresión de CPT1 y HSL (genes involucrados en la oxidación de ácidos grasos), disminuyeron la expresión de HMG-CoA (enzima involucrada en la síntesis de colesterol) y aumenta la CYP7A1 (enzima involucrada en la síntesis de ácidos biliares). ${ }^{39}$ La administración de nanopartículas formuladas a base de lípidos como el oleiletanolamida (OEA) y la oleamida de fenilalaninol (PAO), adicionada con capsaicina en ratones con obesidad inducida, tuvo efectos en el perfil lipídico, el tratamiento con $10 \mathrm{mg}$ OEA $/ \mathrm{kg}+16 \mathrm{mg}$ Capsaicina $/ \mathrm{kg}$ peso disminuyó $(p<0.001)$ los triglicéridos plasmáticos $(71.1 \mathrm{mg} / \mathrm{mL})$, en comparación con el control $(129.5 \mathrm{mg} / \mathrm{mL})$, mientras que la dosis de $12.5 \mathrm{mg}$ PAO + $19.7 \mathrm{mg}$ Capsaicina/kg peso disminuyó $(p<0.05)$ el nivel de colesterol sérico (118 $\mathrm{mg} / \mathrm{dl}$ ) comparado con los controles (234 mg/dl), además, el tratamiento redujo la ingesta diaria de alimento lo cual se relaciona con un efecto de saciedad..$^{40}$ En un estudio realizado en ratas Wistar con obesidad inducida, la administración de una dieta alta en grasas adicionada con $5 \mathrm{mg}$ de un extracto etanólico de C. annuum, tuvo efectos positivos en el perfil lipídico, disminuyó $(p<0.05)$ el colesterol total $(60.67 \mathrm{mg} / \mathrm{dl})$, triglicéridos (36.07 mg/dl), LDL (17.99 mg/dl), VLDL $(7.21$ $\mathrm{mg} / \mathrm{dl})$ y aumentó HDL $(35.47 \mathrm{mg} / \mathrm{dl})$ comparado con el grupo control $(78.27 \mathrm{mg} / \mathrm{dl}, 63.90 \mathrm{mg} / \mathrm{dl}, 36.55 \mathrm{mg} / \mathrm{dl}$, $12.78 \mathrm{mg} / \mathrm{dl}$ y $28.93 \mathrm{mg} / \mathrm{dl}$, respectivamente). ${ }^{41} \mathrm{En}$ ensayos in vitro, reportaron que los extractos etanólicos en concentraciones de $3.61 \mathrm{mg} / \mathrm{mL}$ y $2.71 \mathrm{mg} / \mathrm{mL}$ de especies de $C$. annuum y $C$. frutescens, respectivamente, redujeron la concentración de adipocitos 3T3-L1, además, inhibieron el nivel de lípidos en los adipocitos hasta en un $69 \%$ y $63 \%$, respectivamente; los extractos tuvieron la capacidad de inhibir la actividad de la lipasa pancreática, tratamiento similar con Orlistat, medicamento utilizado en el tratamiento del control de peso. ${ }^{42} \mathrm{La}$ administración de $3.2 \mathrm{~g} / \mathrm{d}$ de una formulación alta en grasas, adicionada con $200 \mathrm{mg} / \mathrm{kg}$ de peso de extracto de capsantina, disminuyó el peso corporal en ratones C57BL/6J con obesidad inducida en un $27.5 \%$, además, la administración de la dieta alta en grasas con capsantina redujo $(p<0.05)$ los niveles séricos de triglicéridos, colesterol y LDL, comparado con aquellos alimentados únicamente con la dieta alta en grasas. ${ }^{43}$ La capsaicina es capaz de unirse a proteínas que activan neuronas sensoriales por medio de estímulos térmicos, vinculándose a la termogénesis; también presenta efecto sobre algunas proteínas implicadas en el metabolismo de lípidos y carbohidratos, lo que permite regular los niveles de hormonas supresoras del apetito ${ }^{44}$, además, tiene la capacidad de aumentar o reducir la expresión de genes como 7- $\alpha$-hidroxilasa (CYP7A1), del transportador 
de ácido biliar ileal (ASBT) y de la proteína ileal de unión al ácido de la bilis (IBABP), implicados en la homeostasis de colesterol, mejorando el metabolismo endógeno de esteroles. ${ }^{45}$

\section{Efectos cardiovasculares}

En un estudio realizado en 42 pacientes, la administración de $4 \mathrm{mg}$ de capsaicina por un periodo de 3 meses, al final de la intervención, resultó en un incremento significativo $(p=0.030)$ en los niveles séricos de colesterol HDL (0.92 mmol/L a $1.00 \mathrm{mmol} / \mathrm{L})$, disminuyeron $(p<0.05)$ los niveles de triglicéridos $(2.62$ $\mathrm{mmol} / \mathrm{L}$ a $2.05 \mathrm{mmol} / \mathrm{L})$, también disminuyó $(p<0.05)$ la actividad de la proteína transportadora de fosfolípidos (PLTP). ${ }^{46}$ El tratamiento de una dieta con $0.006 \%$ de capsaicina en ratas Sprague-Dawley con hipertensión renovascular, redujo la presión sanguínea sistólica y la presión arterial media, además elevó los niveles de eNOS (óxido nítrico sintasa) y p-Akt (Proteína quinasa B) en la aorta torácica, lo que sugiere que la disminución de la presión arterial fue debido a la producción de óxido nítrico a través de la fosforilación de la Akt y eNOS. ${ }^{47}$ Por otro lado, en ratones C57BL/6J alimentados con una dieta rica en grasa, la administración de $0.9 \mathrm{mg}$ de capsaicina $/ \mathrm{kg}$ peso, redujo, y los niveles séricos de colesterol $(p<0.001)$, triglicéridos $(p<0.001)$ y LDL $(p<0.01)$ en un $8.0 \%, 26.5 \%$ y $8.6 \%$ respectivamente, y aumentó los niveles de HDL el $16.5 \%$ comparado con los ratones con dieta alta en grasas sin capsaicina, además, redujo los niveles de malondialdehido y aumentó los niveles de glutatión peroxidasa. ${ }^{48}$ En un estudio in vitro, la aplicación de $40 \mu \mathrm{L}$ de extractos fermentados de Capsicum spp sobre una solución con pulmón de conejo pulverizado, tuvo la capacidad de inhibir la actividad de la enzima convertidora de angiotensina I (enzima se relacionada con la hipertensión) hasta $71.04 \%$, comparado con un extracto crudo, el cual inhibió el 54.29 \%. ${ }^{49}$ La aplicación tópica de una crema adicionada con $0.75 \mathrm{~g}$ de capsaicina $/ \mathrm{kg}$ peso en ratas Wistar con hipoestrogenismo y obesidad inducida, mejoró el perfil lipídico reduciendo el LDL $(p<0.01)$ y triglicéridos $(p<0.001)$, redujo la presión sistólica y diastólica $(p<0.05)$, además, la combinación con ejercicio redujo $(p<0.001)$ los niveles de óxido nítrico y malondialdehido y aumentó los niveles de glutatión $(p<0.05)$ y superóxido dismutasa $(p<0.01)$ comparado con el grupo sedentario. ${ }^{50}$ Los estudios demuestran que los efectos de la capsaicina a nivel endotelial, se obtuvieron a través de la activación del canal iónico TRPV $1^{51}$, lo que permite mejorar el metabolismo energético ${ }^{52}$; además aumentar la expresión de proteínas involucradas en el metabolismo lipídico ${ }^{53}$ y generar un incremento en la fosforilación de PKA (proteína quinasa A), que promueve la producción de óxido nítrico en arterias mesentéricas, reduciendo la presión arterial y mejorando la función vascular. ${ }^{51,47}$ La capsaicina, incrementa los niveles de UCP2, (proteína vinculada a la protección contra el daño causado por especies reactivas de oxígeno en células endoteliales)..$^{53}$

\section{Efecto antidiabético}

La ingesta de $0.01 \%$ y $0.02 \%$ de capsaicina durante 6 semanas en ratones C57BL/6J (ob/ob) con obesidad y diabetes, inhibió el incremento de la glucosa en sangre y de la insulina en ayuno, así como una disminución en los niveles de glucosa. ${ }^{54} \mathrm{La}$ administración de $6 \mathrm{mg}$ de capsaicina y otra de capsiato/kg de peso en ratas Sprague-Dawley con diabetes inducida con estreptozotocina, disminuyó los niveles de glucosa en ayunas ( $18.4 \%$ y $4.9 \%$, respectivamente) en 4 semanas de tratamiento. También disminuyeron los niveles de la proteína sérica glicosilada, los niveles de insulina aumentaron el $50.5 \%$ y $12.2 \%$, respectivamente, al igual que los niveles de glucógeno hepático (66 \% y 16.4 $\%$, respectivamente). ${ }^{55} \mathrm{La}$ administración de $6 \mathrm{mg}$ de capsaicina/kg peso en ratas con diabetes inducida redujo $(p<0.01)$ un $61.9 \%$ los niveles de glucosa en sangre y mostró niveles de insulina de $22.24 \mu \mathrm{lU} / \mathrm{mL}$, mientras que en conejos con diabetes inducida, la misma dosis redujo $(p<0.01)$ los niveles de glucosa en sangre un $54 \%$ comparados con los grupos control. ${ }^{56}$ Otra investigación reportó que el tratamiento de una fórmula alimentaria (con $0.01 \%$ de capsaicina), administrada a ratones $C 57 B L / K s J-(d b / d b)$ con diabetes tipo 2 durante 7 semanas, suprimió el aumento de los niveles de glucosa en sangre en ayunas a partir de la tercera semana de intervención, restauró la tolerancia oral a la glucosa y mejoró la sensibilidad a la insulina. ${ }^{57}$ Por otro lado, la administración de $200 \mathrm{mg} / \mathrm{kg}$ peso de extractos de semillas de $C$. annuum en ratones C57BL/KsJ con diabetes, redujo significativamente $(p<0.001)$ los niveles de glucosa en ayunas, glucosa posprandial (120 min después de la administración), insulina y la hemoglobina glicosilada (HbA1c) el $21 \%, 30 \%, 29 \%$ y $22 \%$, respectivamente. ${ }^{58}$ Los estudios señalan que el efecto antidiabético se atribuye a que la capsaicina ayuda a mejorar la acumulación de ácido tauro- $\beta$-muricolato a nivel intestinal, antagonista del receptor fernesoide $X$ (FXR), el cual actúa regulando el metabolismo de ácidos biliares, reduciendo la gluconeogénesis, y en conjunto mejoran la homeostasis de la glucosa ${ }^{57}$, mediante la activación del receptor TRPV1 y al incremento de expresión de genes implicados en el metabolismo de 
glúcidos en hígado y páncreas, por lo que hay menor absorción de azúcares en el intestino, que posteriormente serán excretados. ${ }^{55}$ A su vez, los extractos de semillas de $C$. annuum aumentan la fosforilación de AMPK (Proteína implicada en el balance energético) y FOXO1 (factor de transcripción que aumenta la síntesis de glucosa en el hígado), que es un mecanismo importante en la mejora de los niveles de glucosa en ayuno y la sensibilidad a la insulina. ${ }^{58}$

\section{Efectos gastrointestinales}

La ingesta de una dieta estándar adicionada con $0.01 \%$ y $0.02 \%$ de capsaicina durante 6 semanas en ratones C57BL/6J, tuvo efectos en la composición de la microbiota intestinal, ya que incrementaron los niveles de Roseburia (bacteria que ayuda a digerir los carbohidratos) y disminuyeron los Bacteroides y los Parabacteroides (bacterias gram negativas que causan infecciones). ${ }^{54}$ En un estudio similar, el tratamiento con $10 \mathrm{mg} / \mathrm{kg}$ con extractos de $C$. annuum, en ratones con obesidad inducida, tuvo efectos en la estructura de la microbiota ya que afectó la relación de bacterias, aumentó Firmicutes y disminuyó Bacteroidetes. ${ }^{59}$ En ratas Wistar con gastritis inducida con ácido acetilsalicílico, la administración oral de $2 \mathrm{mg} / \mathrm{kg}$ peso de capsaicina durante $15 \mathrm{~d}$, reguló la expresión de moléculas pro inflamatorias en la mucosa gástrica (TNF$\alpha$, IL-1B, IL-6 y COX-2), los tejidos gástricos fueron comparables con las ratas sanas aportando $61.1 \%$ de protección. ${ }^{60}$ En un estudio in vitro sobre Helicobacter pylori (bacteria estrechamente relacionada con infecciones gastrointestinales), la capsaicina (62.5 $\mu \mathrm{g} / \mathrm{mL}$ ) tuvo una zona de inhibición de $15 \mathrm{~mm} .^{61} \mathrm{La}$ administración de $10 \mathrm{mg}$ de capsaicina/Kg peso inhibió la formación de úlceras en el antro gástrico hasta en un $50 \%$ en ratones con lesiones inducidas con indometacina. ${ }^{62}$ Como se mencionó anteriormente, la capsaicina activa el receptor TRPV1, expresado por neuronas nociceptivas aferentes, que a nivel gastrointestinal estimula la producción de mucosa gástrica ${ }^{63}$ y la activación de dicho receptor contribuye a la modificación del microbiota intestinal favoreciendo los efectos protectores. ${ }^{54}$

\section{Efecto anticarcinogénico}

En un estudio con 50 canes con diferentes diagnósticos de tumores, la administración de un suplemento alimenticio con extractos de capsaicina de $C$. chinense, disminuyó del $5 \%$ al 50 \% el tamaño del tumor en 15 de ellos, por lo que los efectos favorables de los extractos pudieron inhibir el crecimiento del tumor, o incluso inducir su reabsorción. ${ }^{64}$ En un estudio con perros con cáncer hepático primario, la ingesta de un suplemento alimenticio con capsaicina de $C$. chinense, en dosis de $6.25 \mu \mathrm{g} / 10 \mathrm{~kg}$, redujeron o detuvieron el crecimiento y tamaño de los tumores. ${ }^{65}$ Los extractos metanólicos de capsaicina (Capsicum spp.) utilizados en estudios in vitro, inhibieron $(p<0.00001)$ la actividad proliferativa (20.46 $\mu \mathrm{M}$ en $72 \mathrm{~h}$ ) al $100 \%$ de las células de adenocarcinoma esofágico (OE19), y el $90 \%$ de las células de adenocarcinoma de colon (Caco-2); además los extractos indujeron la apoptosis y regularon los niveles de proteína anti apoptótica (Bcl-2) e incrementaron los niveles de proteína apoptótica (p53), comparados con células fibroblásticas normales. ${ }^{66}$ Por otro lado, en un estudio in vitro, la aplicación de 1000 $\mathrm{mg} / \mathrm{mL}$ de extractos metanólicos de C. Annuum redujeron el $20 \%$ la supervivencia de células de cáncer de próstata (PC3) y la misma concentración con extractos de soxhlet por metanol, redujeron a menos el $20 \%$ el contenido de células. ${ }^{37}$ En otro estudio, la aplicación de extractos de $C$. annuum en cultivos de la línea celular de cáncer de próstata DU147, disminuyó $(p<0.001)$ la proliferación celular en concentraciones de $7 \times 10 \mu \mathrm{g} / \mathrm{mL}$ comparada con las células sin los extractos, mientras que la aplicación de $7 \times 10^{-4} \mu \mathrm{g} / \mathrm{mL}$ de los extractos disminuyó $(p<0.01)$ y la proliferación de células de carcinoma colorrectal HCT116 y disminuyó $(p<0.003)$ células de cáncer de mama MCF7 en 48 h de incubación. ${ }^{67}$ Los estudios reportan que el efecto antiproliferativo de la capsaicina, se relaciona con la producción de hidroperóxido, o con la inhibición de procesos enzimáticos en el sistema de transporte de electrones de la membrana plasmática mitocondrial ${ }^{68}$, direccionando el flujo de electrones que libera oxígeno reactivo en exceso, generando daños estructurales y funcionales, así como apoptosis de las células cancerígenas. ${ }^{65}$ La capacidad de la capsaicina de inhibir el crecimiento de células cancerosas, se relaciona con la detención del ciclo celular G1 (fase de inicio de división celular) ${ }^{69}$, por lo cual la capsaicina puede inducir estrés en las células afectadas y eventualmente conducir a la apoptosis. ${ }^{70}$

\section{Conclusiones}

Las especies del género Capsicum además de ser usado ampliamente en la gastronomía a nivel mundial, debido a las cualidades sensoriales, los estudios científicos evidencian que este fruto tiene gran relevancia en el área de la salud, ya que tiene efectos benéficos a nivel biológico por sus compuestos bioactivos como los fenoles, o el más característico en este fruto, la capsaicina, los cuales tienen la capacidad 
de modificar la expresión de moléculas proinflamatorias, mejorar la sensibilidad de leptina y grelina (hormonas involucradas en los procesos de hambre-saciedad) y regulan la expresión de enzimas que participan en la síntesis de lípidos.

\section{Referencias}

[1] León, J. Botánica de los cultivos tropicales. Tercera edición. Costa Rica: Ed. Agroamérica; 2000. 522 Pp.

[2] Bosland, P. and Votava, E. Peppers: Vegetable and spice Capsicums. Crop production science in horticulture. Second edition. New York: Ed. CABI; 2012. 230 Pp.

[3] Castañón, G., Latournerie, L., Lesher, J. M., de-la-Cruz, E. y Mendoza, M. Identificación de variables para caracterizar morfológicamente colectas de chile (Capsicum spp.) en Tabasco, México. Universidad y Ciencia. 2010; 26(3): 225-234.

[4] Zhang, J., Yang, R., Chen, R., Li, Y. C., Peng, Y., and Wen, X. Geographical origin discrimination of pepper (Capsicum annuum L.) based on multi-elemental concentrations combined with chemometrics. Food Science and Biotechnology. 2019; 28(6): 16271635 .

[5] Namesny, A. Pimientos (Segunda edición). Compendios de Horticultura 16. España: Ediciones de horticultura, S.L. Reus; 2006. 254 Pp.

[6] DeWitt, D. and Bosland, P. The complete chili pepper book: A gardener's guide to choosing, growing, preserving and cooking. USA/ England: Ed. Timber press; 2009. 336 Pp.

[7] Food and agriculture organization of the United Nations. FAOSTAT. Food and agriculture organization of the United Nations Crops; 2018 [En línea]. [Consultado 12 de agosto de 2020] Disponible en: http://www.fao.org/faostat/en/\#data/QC

[8] Barbero, G. F., Liazid, A., Azaroual, L., Palma, M., and Barroso, C. G. Capsaicinoid contents in peppers and pepper-related spicy foods. International Journal of Food Properties. 2016; 19(3): 485-493.

[9] Castellón-Martínez, É., Chávez-Servia, J. L., Carrillo-Rodríguez, J. C. y Vera-Guzmán, A. M. Preferencias de consumo de chiles (Capsicum annuum L.) nativos en los valles centrales de Oaxaca, México. Revista Fitotecnia Mexicana. 2012; 35(SPE5): 27-35.

[10] Yánez, P., Balseca, D., Rivadeneira, L. y Larenas, C. Características morfológicas y de concentración de capsaicina en cinco especies nativas del género Capsicum cultivadas en Ecuador. La Granja. Revista de Ciencias de la Vida. 2015; 22(2): 12-32.

[11] Thuphairo, K., Sornchan, P., and Suttisansanee, U. Bioactive compounds, antioxidant activity and inhibition of key enzymes relevant to Alzheimer's disease from sweet pepper (Capsicum annuum) extracts. Preventive nutrition and food science. 2019; 24(3), 327.

[12] Hernández-Verdugo, S., Porras, F., Pacheco-Olvera, A., LópezEspaña, R. G., Villarreal-Romero, M., Parra-Terraza, S. y OsunaEnciso, T. Caracterización y variación ecográfica de poblaciones de chile (Capsicum annum var. Glabriusculum) silvestre del noroeste de México. Polibotánica. 2012; 33(1): 175-191.

[13] Palacios, S. y García, M. Caracterización morfológica de 93 accesiones de Capsicum spp del banco de germoplasma de la
Universidad Nacional de Colombia Sede Palmira. Acta Agronómica. 2008; 57(4): 247-252.

[14] Ramírez, M., Arcos, G. y Méndez, R. Jaguar: cultivar de chile habanero para México. Revista Mexicana de Ciencias Agrícolas. 2018; 9(2): 487-492.

[15] Medina, E. L., Zabaleta, A. L., Rivero, A. E. G., León, J. M., Anthony, J. y Zapata, L. V. Morfometría de frutos y semillas del “ají mochero" Capsicum chinense Jacq. Ciencia \& Tecnología Agropecuaria. 2020; 21(3): 1-11.

[16] de-la-Cruz-Lázaro, E., Márquez-Quiroz, C., Osorio-Osorio, R., Preciado-Rangel, P. y Márquez-Hernández, C. Caracterización morfológica in situ de chile silvestre Pico de paloma (Capsicum frutescens) en Tabasco, México. Acta Universitaria. 2017; 27(2): 1016.

[17] Escalera-Ordaz, A. K., Guillén-Andrade, H., Lara-Chávez, M. B. N., Lemus-Flores, C., Rodríguez-Carpena, J. G. y Valdivia-Bernal, R. Caracterización de variedades cultivadas de Capsicum pubescens en Michoacán, México. Revista Mexicana de Ciencias Agrícolas. 2019; (23): 239-251.

[18] Bae, H., Jayaprakasha, G. K., Crosby, K., Yoo, K. S., Leskovar, D. I., Jifon, J., and Patil, B.S. Ascorbic acid, capsaicinoid, and flavonoid aglycone concentrations as a function of fruit maturity stage in greenhouse-grown peppers. Journal of Food Composition and Analysis. 2014; 33(2): 195-202.

[19] Rochín-Wong, C. S., Gámez-Meza, N., Montoya-Ballesteros, L. C. y Medina-Juárez, L. A. Efecto de los procesos de secado y encurtido sobre la capacidad antioxidante de los fitoquímicos del chiltepín (Capsicum annuum L. var. glabriusculum). Revista mexicana de ingeniería química. 2013; 12(2): 227-239.

[20] Carvalho, A. V., de-Andrade-Mattietto, R., de-Oliveira-Rios, A., deAlmeida-Maciel, R., Moresco, K. S., and de-Souza-Oliveira, T. C. Bioactive compounds and antioxidant activity of pepper (Capsicum sp.) genotypes. Journal of Food Science and Technology. 2015; 52(11): 7457-7464.

[21] Gurnani, N., Gupta, M., Darshana, M. D., and Mehta, B. K. Chemical composition, total phenolic and flavonoid contents, and in vitro antimicrobial and antioxidant activities of crude extracts from red chilli seeds (Capsicum frutescens L.). Journal of Taibah University of Science. 2016; 10(4): 462-470.

[22] Cervantes-Paz, B., Yahia, E. M., Ornelas-Paz, J. de J., VictoriaCampos, C. I., Ibarra-Junquera, V., Pérez-Martínez, J. D., and Escalante-Minakata, P. Antioxidant activity and content of chlorophylls and carotenoids in raw and heat-processed Jalapeño peppers at intermediate stages of ripening. Food Chemistry. 2014; (146): 188-196.

[23] Rodríguez-Burruezo, A., González-Mas, M. D. C., and Nuez, F. Carotenoid composition and vitamin A value in ají (Capsicum baccatum L.) and rocoto (C. pubescens R. \& P.), 2 pepper species from the Andean region. Journal of Food Science. 2010; 75(8): 446453 .

[24] Sricharoen, P., Lamaiphan, N., Patthawaro, P., Limchoowong, N., Techawongstien, S., and Chanthai, S. Phytochemicals in Capsicum oleoresin from different varieties of hot chilli peppers with their antidiabetic and antioxidant activities due to some phenolic compounds. Ultrasonics Sonochemistry. 2017; (38): 629-639.

[25] Zimmer, A. R., Leonardi, B., Miron, D., Schapoval, E., de-Oliveira, J. R., and Gosmann, G. Antioxidant and anti-inflammatory properties 
of Capsicum baccatum: from traditional use to scientific approach. Journal of Ethnopharmacology. 2012; 139(1): 228-233.

[26] Pugliese, A., Loizzo, M. R., Tundis, R., O’Callaghan, Y., Galvin, K., Menichini, F., and O'Brien, N. The effect of domestic processing on the content and bioaccessibility of carotenoids from chili peppers (Capsicum species). Food Chemistry. 2013; 141(3): 2606-2613.

[27] Howard, L. R., Talcott, S. T., Brenes, C. H., and Villalon, B. Changes in phytochemical and antioxidant activity of selected pepper cultivars (Capsicum species) as influenced by maturity. Journal of Agricultural and Food Chemistry. 2000; 48(5): 1713-1720.

[28] Rivas, M. A., Vignale, N. D., Ordóñez, R. M., Zampini, I. C., Alberto, M. R., Sayago, J. E. e Isla, M. I. Nutraceutical properties and toxicity studies of flour obtained from Capsicum pubescens fruits and its comparison with "Locoto" commercial powder. Food and Nutrition Science. 2014; (5): 715-724.

[29] da-Silveira-Agostini-Costa, T., da-Silva-Gomes, I., de-Melo, L. A. M. P., Reifschneider, F. J. B., and da-Costa-Ribeiro, C. S. Carotenoid and total vitamin $\mathrm{C}$ content of peppers from selected Brazilian cultivars. Journal of Food Composition and Analysis. 2017; (57): 7379.

[30] Baas-Espinola, F. M., Castro-Concha, L. A., Vázquez-Flota, F. A., and Miranda-Ham, M. L. Capsaicin synthesis requires in situ phenylalanine and valine formation in in vitro maintained placentas from Capsicum chinense. Molecules. 2016; 21(6): 799.

[31 Giuffrida, D., Dugo, P., Torre, G., Bignardi, C., Cavazza, A., Corradini, C., and Dugo, G. Characterization of 12 Capsicum varieties by evaluation of their carotenoid profile and pungency determination. Food Chemistry. 2013; (140): 794-802.

[32] Wang, X., Yu, L., Li, F., Zhang, G., Zhou, W., and Jiang, X. Synthesis of amide derivatives containing capsaicin and their antioxidant and antibacterial activities. Journal of Food Biochemistry. 2019; 43(12): 1-9.

[33] Boiko, Y. A., Kravchenko, I. A., Shandra, A. A., and Boiko, I. A. Extraction, identification and anti-inflammatory activity of carotenoids out of Capsicum Anuum L. Journal of Herbmed Pharmacology. 2016; 6(1): 10-15.

[34] Alabi, A., Ajayi, A. M., Olooto, W. E., Emegoakor, C., Oladunjoye, O., and Obikoya, Y. Antinociceptive and anti-inflammatory properties of a polyherbal extract of Plumbago zeylinica and Capsicum frutescens in rodents. African Journal of Biomedical Research. 2017; 20(3): 277-285.

[35] Prathoshni, S. M., Anitha, R., and Lakshmi, T. The effect of Capsicum oleoresin on nitric oxide production and nitric oxide synthase gene expression in macrophage cell line. Pharmacognosy Research. 2018; 10(4): 343.

[36] Qiao, G. H., Wenxin, D., Zhigang, X., Sami, R., Khojah, E., and Amanullah, S. Antioxidant and anti-inflammatory capacities of pepper tissues. Italian Journal of Food Science. 2020; 32(2): 265-274.

[37] Della-Valle, A., Dimmito, M. P., Zengin, G., Pieretti, S., Mollica, A., Locatelli, M., ..., and Baloglu, M. C. Exploring the nutraceutical potential of dried pepper Capsicum annuum L. on market from Altino in Abruzzo Region. Antioxidants. 2020; 9(5): 400.

[38] Allemand, A., Leonardi, B. F., Zimmer, A. R., Moreno, S., Romao, P. R. T., and Gosmann, G. Red pepper (Capsicum baccatum) extracts present anti-inflammatory effects in vivo and inhibit the production of TNF- $\alpha$ and NO in vitro. Journal of medicinal food. 2016; 19(8): 759-767.

[39] Liu, L., Ding, C., Tian, M., Yi, D., Wang, J., Zhao, J., ..., and Wang, C. Fermentation improves the potentiality of capsicum in decreasing high-fat diet-induced obesity in C57BL/6 mice by modulating lipid metabolism and hormone response. Food Research International. 2019; 124: 49-60.

[40] Lacatusu, I., Badea, N., Udeanu, D., Coc, L., Pop, A., Negut, C. C., ..., and Meghea, A. Improved anti-obesity effect of herbal active and endogenous lipids co-loaded lipid nanocarriers: Preparation, in vitro and in vivo evaluation. Materials Science and Engineering. 2019; 99 : $12-24$.

[41] Al-Jumayi, H. A., Elhendy, H. A., and Darwish, A. M. Biological effects of red chili pepper (Capsicum annuum) consumption on high fat diet female albino rats. Pakistan Journal of Biological Sciences: PJBS. 2020; 23(2): 150-158.

[42] Huei, C. S., Azlan, A., Ismail, A., Shafie, N. H., and Sultana, S. Antioxidant and anti-obesity properties of local chilies varieties in Malaysia. Journal of Food Science and Technology. 2020; 57(10): 3677-3687.

[43] Wu, T., Gao, Y., Hao, J., Geng, J., Zhang, J., Yin, J., ..., and Zhang, M. Capsanthin extract prevents obesity, reduces serum TMAO levels and modulates the gut microbiota composition in high-fat-diet induced obese C57BL/6J mice. Food Research International. 2020; 128: 108774 .

[44] Joo, J. I., Kim, D. H., Choi, J. W., and Yun, J. W. Proteomic analysis for antiobesity potential of capsaicin on white adipose tissue in rats fed with a high fat diet. Journal of Proteome Research. 2010; 9(6): 2977-2987.

[45] Zhang, L., Fang, G., Zheng, L., Chen, Z., and Liu, X. Hypocholesterolemic effect of capsaicinoids in rats fed diets with or without cholesterol. Journal of Agricultural and Food Chemistry. 2013; 61(18): 4287-4293.

[46] Qin, Y., Ran, L., Wang, J., Yu, L., Lang, H. D., Wang, X. L., ..., and Zhu, J. D. Capsaicin supplementation improved risk factors of coronary heart disease in individuals with low HDL-C levels. Nutrients. 2017; 9(9): 1037.

[47] Segawa, Y., Hashimoto, H., Maruyama, S., Shintani, M., Ohno, H., Nakai, Y., $\ldots$ and Kurihara, N. Dietary capsaicin-mediated attenuation of hypertension in a rat model of renovascular hypertension. Clinical and Experimental Hypertension. 2019; 42(4): 352-359.

[48] Li, W., Yang, H., and Lu, Y. Capsaicin alleviates lipid metabolism disorder in high beef fat-fed mice. Journal of Functional Foods. 2019; 60: 103444.

[49] Yeon, S. J., Kim, J. H., Cho, W. Y., Kim, S. K., Seo, H. G., and Lee, C. H. In Vitro studies of fermented korean chung-yang hot pepper phenolics as inhibitors of key enzymes relevant to hypertension and diabetes. Foods. 2019; 8(10): 498.

[50] de-Lourdes-Medina-Contreras, J. M., Mailloux-Salinas, P., ColadoVelazquez, J. I., Gómez-Viquez, N. L. G. V., Velázquez-Espejel, R., del-Carmen-Susunaga-Notario, A., and Bravo, G. Topical capsaicin cream with moderate exercise protects against hepatic steatosis, dyslipidemia and increased blood pressure in hypoestrogenic obese rats. Journal of the Science of Food and Agriculture. 2020; 100(7): 3212-3219. 
[51] Yang, D., Luo, Z., Ma, S., Wong, W. T., Ma, L., Zhong, J., ..., and Liu, D. Activation of TRPV1 by dietary capsaicin improves endothelium-dependent vasorelaxation and prevents hypertension. Cell Metabolism. 2010; 12(2): 130-141.

[52] Luo, Z., Ma, L., Zhao, Z., He, H., Yang, D., Feng, X., ..., and Liu, D. TRPV1 activation improves exercise endurance and energy metabolism through PGC-1 $\alpha$ upregulation in mice. Cell Research. 2012; 22(3): 551-564.

[53] Xiong, S., Wang, P., Ma, L., Gao, P., Gong, L., Li, L., ..., and Chen, J. Ameliorating endothelial mitochondrial dysfunction restores coronary function via transient receptor potential vanilloid 1mediated protein kinase $\mathrm{A} /$ uncoupling protein 2 pathway. Hypertension. 2016; 67(2): 451-460.

[54] Song, J. X., Ren, H., Gao, Y. F., Lee, C. Y., Li, S. F., Zhang, F., ..., and Chen, H. Dietary capsaicin improves glucose homeostasis and alters the gut microbiota in obese diabetic ob/ob mice. Frontiers in Physiology. 2017; 8: 602.

[55] Zhang, S., Ma, X., Zhang, L., Sun, H., and Liu, X. Capsaicin reduces blood glucose by increasing insulin levels and glycogen content better than capsiate in streptozotocin-induced diabetic rats. Journal of Agricultural and Food Chemistry. 2017; 65(11): 2323-2330.

[56] Lagisetty, U., Mohammed, H., and Ramaiah, S. Effect of capsaicin on pharmacodynamic and pharmacokinetics of gliclazide in animal models with diabetes. Pharmacognosy Research. 2018; 10: 437-41.

[57] Hui, S., Liu, Y., Chen, M., Wang, X., Lang, H., Zhou, M., ..., and Mi, M. Capsaicin improves glucose tolerance and insulin sensitivity through modulation of the gut microbiota-bile acid-FXR axis in type 2 diabetic db/db mice. Molecular Nutrition \& Food Research. 2019; 63(23): 1900608 .

[58] Kim, H. K., Jeong, J., Kang, E. Y., and Go, G. W. Red Pepper (Capsicum annuum L.) Seed extract improves glycemic control by inhibiting hepatic gluconeogenesis via phosphorylation of FOXO1 and AMPK in obese diabetic db/db mice. Nutrients. 2020; 12(9): 2546 .

[59] Sinisgalli, C., Vezza, T., Diez-Echave, P., Ostuni, A., Faraone, I., Hidalgo-Garcia, L., ..., and Nogales, A. R. The beneficial effects of red sun-dried Capsicum annuum L. cv Senise extract with antioxidant properties in experimental obesity are associated with modulation of the intestinal microbiota. Molecular Nutrition \& Food Research. $2020 ; 2000812$.

[60] Mendivil, E. J., Sandoval-Rodriguez, A., Meza-Ríos, A., ZuñigaRamos, L., Dominguez-Rosales, A., Vazquez-Del-Mercado, M., ..., and Armendariz-Borunda, J. Capsaicin induces a protective effect on gastric mucosa along with decreased expression of inflammatory molecules in a gastritis model. Journal of Functional Foods. 2019; 59: 345-351.

[61] Tayseer, I., Aburjai, T., Abu-Qatouseh, L., AL-Karabieh, N., Ahmed, W., and Al-Samydai, A. In vitro anti Helicobacter pylori activity of capsaicin. J. Pure Appl. Microbiol. 2020; 14(1): 279-286.

[62] Satoh, H., Akiba, Y., and Urushidani, T. Proton pump inhibitors prevent gastric antral ulcers induced by NSAIDs via activation of capsaicin-sensitive afferent nerves in mice. Digestive Diseases and Sciences. 2020; 65(9): 2580-2594.

[63] Montero, R. D., Cortez, D. F. y Pacheco, E. V. Efecto del Capsicum annum L. (pucunucho, ají mono) en úlcera gástrica experimental inducida en. Revista de Gastroenterología del Perú. 2017; 35(2): 141150
[64] Adaszek, Ł. U. K. A. S. Z., Gadomska, D., Staniec, M., Gołyński, M., Łyp, P., Ziętek, J., ..., and Winiarczyk, S. Clinical assessment of the anti-cancer activity of the capsaicin-containing habanero pepper extract in dogs-preliminary study. Med. Weter. 2017; 73(7): 404411 .

[65] Dębiak, P., Gadomska, D., Śmiech, A., Ziętek, J., Łyp, P., ŁojszczykSzczepaniak, A. N. N. A., and Adaszek, Ł. Effectiveness of capsaicin containing dried habanero pepper extract in the treatment of primary hepatic cancer in geriatric dogs. Medycyna Weterynaryjna. 2018; 74(12): 765-771.

[66] Lavorgna, M., Orlo, E., Nugnes, R., Piscitelli, C., Russo, C., and Isidori, M. Capsaicin in hot chili peppers: in vitro evaluation of its antiradical, antiproliferative and apoptotic activities. Plant Foods for Human Nutrition. 2019; 74(2): 164-170.

[67] Hatzidaki, E., Papadimitriou, M., and Papasotiriou, I. Evaluation of the anti-proliferative effects of a green tea and Capsicum powder extract in cancer cell lines. Journal of Cancer Therapy. 2020; 11(2): 44-54.

[68] Hail-Jr, N. and Lotan, R. Examining the role of mitochondrial respiration in vanilloid-induced apoptosis. Journal of the National Cancer Institute. 2002; 94(17): 1281-1292

[69] Zhang, J., Nagasaki, M., Tanaka, Y., and Morikawa, S. Capsaicin inhibits growth of adult T-cell leukemia cells. Leukemia Research. 2003; 27(3): 275-283.

[70] Sarpras, M., Chhapekar, S. S., Ahmad, I., Abraham, S. K., and Ramchiary, N. Analysis of bioactive components in Ghost chili (Capsicum chinense) for antioxidant, genotoxic, and apoptotic effects in mice. Drug and Chemical Toxicology. 2018; 43(2): 182-10. 